\title{
ORIGINAL
}

\section{LA SATISFACCIÓN DE LAS NECESIDADES DE LAS PERSONAS DEPENDIENTES DE 60 AÑOS Y MÁS SEGÚN PROVEEDOR DE CUIDADO. ESPAÑA, 2008 (*)}

\author{
Jesús Rogero-García (1) y Karim Ahmed-Mohamed (2)
}

(1) Universidad Autónoma de Madrid, Departamento de Sociología.

(2) Centro de Investigaciones Sociológicas.

(*) Trabajo financiado parcialmente por el Ministerio de Ciencia e Innovación (proyecto de investigación "Los usos del tiempo en España e Iberoamérica: los tiempos del cuidado”, CSO2008-04747).

\section{RESUMEN}

Fundamento: A pesar de su importancia para el diseño de políticas públicas, son escasos los estudios sobre el grado en que el cuidado que reciben las personas mayores satisface sus necesidades. Los objetivos de este trabajo son: conocer la prevalencia de personas de 60 y más años que reciben atención y que tienen necesidades de cuidado no satisfechas; y establecer qué proveedor de cuidado (familiar, privado - empleadas de hogar- o público -servicios sociales-) tiene más probabilidades de satisfacer dichas necesidades.

Métodos: Se utilizó una muestra de 3.718 personas de 60 y más años no institucionalizadas de la Encuesta de Discapacidad, Autonomía personal y situaciones de Dependencia 2008. Se realizaron tablas de contingencia para conocer la distribución de las necesidades de cuidado no satisfechas y se construyó un modelo de regresión logística binaria para identificar los factores asociados con las necesidades no satisfechas.

Resultados: De las personas de 60 y más años que recibían atención, no satisficieron sus necesidades de cuidado 1039 (29\%). Respecto a quienes recibieron únicamente cuidado familiar, quienes recibieron sólo cuidado de servicios sociales tuvieron menos probabilidades de tener satisfechas sus necesidades $(\mathrm{OR}=1,932 \mathrm{sig} .=0,001)$ y quienes recibieron únicamente cuidado privado tuvieron más probabilidades $(\mathrm{OR}=0,673$ sig. $=0,015)$

Conclusiones: Quienes recibieron exclusivamente cuidado privado mostraron más probabilidades de tener satisfechas sus necesidades que quienes recibieron cualquier otra combinación de cuidado. Recibir únicamente atención de servicios sociales se relaciona con una mayor probabilidad de tener necesidades de cuidado insatisfechas, en relación con quienes reciben cuidado familiar o cuidado privado.

Palabras clave: Anciano. Anciano frágil. Personas con discapacidad. Cuidadores familiares. Servicios de atención de salud a domicilio. Evaluación de necesidades. España.

\section{ABSTRACT \\ Satisfaction of Needs of Disabled People 60 and over According to Care Provider. Spain, 2008}

Background: In spite of its importance for policy making, there have been few studies examining the satisfaction of care needs by the different care providers. The aims of this paper are: (1) to estimate the prevalence of dissatisfaction of care needs among people older than 59 years receiving care; and (2) to identify what care provider (family care, private care - paid care- or public care) has a higher probability of satisfying the needs of this population.

Methods: This study is based on a sample of 3718 non-institutionalized people older than 59 years from the Survey on Disability, Personal Autonomy and Dependency Situations 2008. The distribution of unmet care needs among different groups of elderly people was cross-tabulated, and a regression logistic model was used to identify factors related to dissatisfaction of care needs.

Results: 1,039 (29\%) people older than 59 years had dissatisfied care needs. Compared to those receiving only family care, people receiving only public care were more likely to have dissatisfied needs $(\mathrm{OR}=1.932$ sig. $=0,001)$, and people receiving only paid care were less likely to have dissatisfied needs $(\mathrm{OR}=0.673 \mathrm{sig} .=0.015)$.

Conclusions: People receiving only paid care had a higher probability of having their needs satisfied than any other care combination. Those receiving only public care had a lower probability of having their needs satisfied than people receiving family care or paid care.

Key Words: Aged. Frail elderly. Disabled persons. Caregivers. Family Caregivers. Home care services. Needs assessment. Spain.

\author{
Correspondencia: \\ Jesús Rogero García \\ Universidad Autónoma de Madrid \\ Facultad de Ciencias Económicas y Empresariales \\ C. Francisco Tomás y Valiente, 5 \\ Campus de Cantoblanco \\ 28049 Madrid \\ jesus.rogero@uam.es
}




\section{INTRODUCCIÓN}

En los últimos años se ha producido un intenso crecimiento de los estudios sobre cuidado a adultos dependientes, especialmente en relación con personas mayores. Gran parte de estas investigaciones han prestado atención al impacto del cuidado en diferentes dimensiones de la vida de los cuidadores familiares, como la salud, la economía o las relaciones sociales. Otra buena parte de las investigaciones en este campo han estudiado la distribución de los diferentes proveedores de cuidado (formal, informal y combinado) entre la población de diferentes países. En la mayoría de ellos se ha observado de manera reiterada que el cuidado familiar está considerablemente más extendido que el resto de tipos de atención ${ }^{1,2}$. A pesar de los numerosos estudios sobre cuidado a personas dependientes, la literatura sobre necesidades de cuidado no satisfechas es limitada, y la existente presenta lagunas importantes ${ }^{3}$. Entre otras carencias, apenas hay estudios que comparen el efecto que tienen los diferentes proveedores de cuidado en la satisfacción de las necesidades de las personas dependientes. Esta escasez de estudios es especialmente llamativa en el contexto de implementación de la Ley de Dependencia $(39 / 2006)^{4}$.

Las personas se encuentran en situación de dependencia cuando, debido a la falta o pérdida de autonomía, requieren ayudas personales para realizar las denominadas actividades de la vida cotidiana (AVD) ${ }^{5}$. Estas actividades se dividen generalmente en tres categorías: físicas, que se refieren a las tareas básicas de autocuidado; instrumentales, relativas con actividades cotidianas más complejas; y de movilidad, que pueden estar incluidas en las dos primeras categorías $^{6}$. Las fuentes de cuidado más frecuentes en España son el cuidado familiar, el cuidado privado (procedente fundamentalmente de empleadas de hogar) y el cuidado provisto desde las administraciones públicas. Cada agente proveedor de cuidado tiene efectos diferenciados en las personas dependientes, debido a que su eficacia en la realización de las tareas y en el apoyo emocional no es la misma ${ }^{7}$.

El cuidado familiar implica generalmente apoyo físico, emocional e informacional ${ }^{8,9}$. Los cuidadores familiares realizan una gran variedad de actividades, como administrar medicación, ayudar en el aseo diario, preparar comidas o gestionar los servicios de salud de las personas dependientes. Generalmente, los cuidadores remunerados (con dinero público o privado) asumen una menor diversidad de actividades, especialmente en el caso de aquellos servicios financiados desde las administraciones públicas. Las características que definen el cuidado formal son: (a) que se produce a cambio de un salario, (b) que se realiza por un profesional, o (c) que es provisto a través de una organización (pública, privada o sin ánimo de lucro). El cuidado formal aparece con mayor frecuencia cuando las necesidades de cuidado son mayores ${ }^{10}$. En España, el recurso de las empleadas de hogar como cuidadoras principales, generalmente inmigrantes, ha experimentado una expansión en los últimos años ${ }^{11}$. El cuidado público es, en comparación con el cuidado familiar y privado, el que tiene una menor presencia entre las personas dependientes ${ }^{12}$.

Se considera que las necesidades de cuidado no se cubren cuando la asistencia de otra/s persona/s es inadecuada, insuficiente o inexistente ${ }^{3}$. Los estudios sobre necesidades de cuidado no cubiertas remiten a la relación entre el cuidado que se desarrolla y la satisfacción que produce. Las personas dependientes consideran que sus necesidades de cuidado no están cubiertas cuando no están satisfechas con el cuidado que reciben, de modo que ambos conceptos, necesidad no cubierta e insatisfacción con el cuidado, responden a una realidad similar: la de quienes no reciben el apoyo que necesitan (o así lo consideran). La idea de satisfacción con el cuidado contiene tres elementos: 
las características del cuidado recibido, las preferencias personales y las expectativas sobre el mismo ${ }^{13}$. El cuidado es adecuado cuando sus componentes (tiempo, actividades desempeñadas y actitudes desarrolladas) sirven para satisfacer las necesidades de atención de la persona cuidada.

Las consecuencias negativas de no tener satisfechas las necesidades de apoyo son numerosas e influyen intensamente en la calidad de vida de las personas dependientes. Allen y Mor ${ }^{14}$ hallaron que estas consecuencias negativas eran experimentadas tanto por quienes declararon necesitar ayuda y no recibirla como por quienes declararon no recibir suficiente ayuda. Entre ellas, se ha detectado un aumento de la depresión ${ }^{15}$, de falta de autonomía ${ }^{8}$, de la institucionalización ${ }^{16}$ y de la utilización de servicios de salud (consultas médicas, urgencias y hospitalización $)^{14}$. Los análisis sobre necesidades de cuidado no satisfechas son, por tanto, esenciales para el diseño de políticas públicas eficaces de atención a las personas dependientes. Asimismo, la detección de estas necesidades constituye uno de los principales objetivos de las grandes operaciones estadísticas sobre discapacidad y dependencia en nuestro país ${ }^{17}$. Llama la atención, por tanto, la escasez de estudios sobre esta cuestión no sólo en España, sino también en otros países.

Este trabajo tiene como objetivos: (1) conocer la prevalencia de necesidades de cuidado no satisfechas entre las personas dependientes de 60 y más años que reciben atención personal; y (2) establecer qué proveedor de cuidado (familiar, público o privado) tiene más probabilidades de satisfacer las necesidades de esta población.

\section{SUJETOS Y METODOS}

Se utiliza información de la Encuesta de Discapacidad, Autonomía personal y situaciones de Dependencia 2008 (EDAD), llevada a cabo por el Instituto Nacional de
Estadística español. El objetivo general de esta encuesta es proporcionar una base estadística que permita guiar la promoción de la autonomía personal y la prevención de las situaciones de dependencia ${ }^{17}$. Su muestra es representativa de la población española y de cada una de sus regiones. Contiene información sobre personas con discapacidad que viven en hogares y sobre establecimientos colectivos, en concreto centros de personas mayores, centros de personas con discapacidad menores de 65 años, hospitales psiquiátricos y hospitales geriátricos. Se entrevistaron a un total de 258.187 personas en hogares, de las cuales 22.795 indicaron experimentar alguna discapacidad. La utilización de informantes indirectos se ha demostrado poco fiable en la valoración subjetiva de la situación de las personas con discapaci$\mathrm{dad}^{18}$, de manera que, para este trabajo, se seleccionaron sólo aquellas personas de 60 o más años que respondieron directamente a la pregunta sobre los proveedores de los que recibían cuidado (se excluyeron los informantes indirectos). La información se recogió a través de entrevistas cara a cara que fueron, excepcionalmente, complementadas con entrevistas telefónicas. La información sobre los cuidadores principales proviene de los propios cuidadores. La muestra final estuvo compuesta por 3.718 casos.

Este trabajo utiliza como variable dependiente principal la satisfacción de las necesidades de cuidado de la persona dependiente, que proviene de la pregunta: “¿Considera que la ayuda que recibe de otras personas satisface sus necesidades? Sí / no, es insuficiente". Se trata, por tanto, de una medida global que informa sobre la eficacia general de la atención recibida, pero que no discrimina entre tipos de necesidades ni entre dimensiones de cuidado específicas (instrumental, afectiva, etc.). La variable "proveedor de cuidado" está conformada por 6 categorías: únicamente cuidado familiar; únicamente cuidado privado (remunerado por la familia); únicamente cuidado proveniente de los servicios sociales (al menos en parte 
financiado públicamente); cuidado familiar y privado; cuidado familiar y de servicios sociales; y cuidado provisto por los tres agentes a la vez.

Para el análisis bivariado se escogieron cuatro tipos de variables, cuyas categorías se detallan en la tabla 3: (a) variables relativas a la persona dependiente: sexo, edad, número de discapacidades (sobre una lista de 44 problemas para realizar actividades de la vida diaria) y nivel de estudios; (b) variables relativas al entorno: tamaño del hogar (número de personas que conviven en el hogar), ingresos mensuales del hogar y tamaño del municipio (número de habitantes); (c) variables relativas al cuidador principal (persona que asume la mayor parte de las tareas de cuidado): sexo, relación de parentesco, situación laboral, nivel de estudios, nacionalidad y problemas a raíz del cuidado (dificultades con el propio cuidado, problemas de salud, problemas laborales y problemas de relaciones sociales u ocio); (d) variables relativas al cuidado recibido: lugar de residencia del cuidador, horas de cuidado recibidas (estimadas por las personas dependientes) y tiempo que el cuidador principal lleva prestando los cuidados.

Se utilizaron tablas de contingencia bivariadas para explorar las relaciones básicas entre las variables descriptivas y la satisfacción de necesidades provista por cualquier proveedor de cuidado. Las diferencias entre grupos fueron analizadas utilizando el test de $\mathrm{chi}^{2}$. Asimismo, se llevó a cabo un modelo de regresión logística binaria para identificar los factores asociados con la satisfacción de necesidades provista por cada proveedor de cuidado. Los modelos de regresión logística analizan si una variable dicotómica está asociada estadísticamente a uno o más factores, y predicen la probabilidad de que se produzca un determinado evento bajo ciertas circunstancias ${ }^{19}$. En este modelo se introdujeron, en un primer paso, las variables relativas a la persona dependiente y a su entorno. En un segundo paso fue introducida la variable "proveedor de cuidado" para comprobar si la consideración de este factor mejora sustancialmente la capacidad predictiva del modelo, así como para ver el efecto de cada proveedor de cuidado en la satisfacción de las necesidades de la persona dependiente. No se incluyeron las variables relativas al cuidador principal ni las relativas al cuidado recibido (excepto las horas de cuidado). La mayoría de estas cuestiones fueron respondidas únicamente por aquellos cuyo cuidado era familiar o, en algunos casos, privado, lo que habría introducido un sesgo en la estimación del efecto de la variable "proveedor de cuidado" sobre la probabilidad de tener satisfechas las necesidades. Para medir el ajuste de los modelos se utilizaron la Chi-cuadrado de Pearson y la $\mathrm{R}^{2}$ de Nagelkerke ${ }^{20}$. La multicolinealidad se evaluó a través del factor de tolerancia, y las variables con factor de tolerancia menor que 0,650 fueron excluidas de los modelos. En todos los análisis, los valores de $\mathrm{p}$ se muestran con tres decimales, con los valores menores de 0,001 expresados como $\mathrm{p}<0,001$. El análisis de los datos se realizó con la versión 17 del software SPSS.

\section{RESULTADOS}

La tabla 1 indica las actividades más frecuentes para las que las personas de $60 \mathrm{y}$ más años tienen dificultades importantes, según proveedor de cuidado. Como era de esperar, hay una mayor presencia de dificultades en Actividades Instrumentales de la Vida Diaria (p. e. ocuparse de las tareas de la casa, preparar comidas, etc.) que en Actividades Básicas de la Vida Diaria (por ejemplo realizar los cuidados básicos del cuerpo, andar o moverse dentro de su vivienda) el porcentaje de personas con dificultades es más elevado entre quienes reciben cuidado de más de un proveedor. La tabla 2 muestra el porcentaje de personas dependientes de 60 y más años que recibían cuidado de cada uno de los proveedores identificados, así como el porcentaje de quienes consideraban que el cuidado 


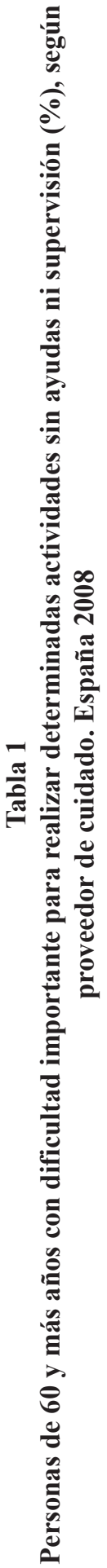

\begin{tabular}{|c|c|c|c|c|c|c|c|c|c|c|c|}
\hline 㞼 & : & @ & ○ी & $8^{2}$ & 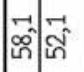 & 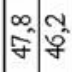 & $\begin{array}{l}n \\
\stackrel{n}{m}\end{array}$ & 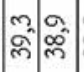 & 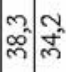 & $\mid \begin{array}{l}y \\
\infty \\
\sim\end{array}$ & : \\
\hline 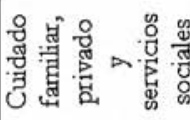 & वू & 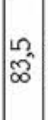 & व. & 2 & 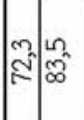 & 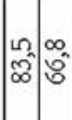 & $\left|\begin{array}{l}\infty \\
\bigcup^{n}\end{array}\right|$ & $\begin{array}{ll}0 & \infty \\
\tilde{n}^{n} & \infty \\
0^{\circ}\end{array}$ & \begin{tabular}{l|l}
$\infty$ & 0 \\
0 \\
0
\end{tabular} & & $\hat{n}$ \\
\hline 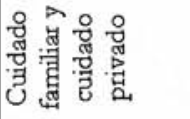 & $\begin{array}{c}\infty \\
\infty \\
\infty \\
\infty\end{array}$ & $\begin{array}{l}\infty \\
\infty \\
\infty \\
\infty\end{array}$ & $\begin{array}{l}a \\
\hat{n}\end{array}$ & స్ & \begin{tabular}{l|l}
$\vec{y}$ & \multicolumn{2}{c}{} \\
\multirow{5}{}{} & in
\end{tabular} & 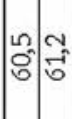 & $\mid \begin{array}{c}-1 \\
\vec{f}\end{array}$ & 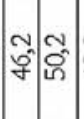 & 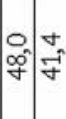 & ले & $\hat{i}$ \\
\hline 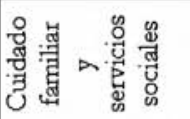 & $\stackrel{\infty}{\infty}$ & $\begin{array}{l}0 \\
0 \\
0 \\
2\end{array}$ & $\stackrel{\cdots}{\approx}$ & స్ & 辣 & $\begin{array}{lll}\infty & 0 \\
\vdots \\
\vdots\end{array}$ & $=$ & 에료 & \begin{tabular}{lll}
2 & \multicolumn{2}{c}{} \\
$n^{2}$ & \multirow{5}{*}{}
\end{tabular} & - & 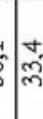 \\
\hline 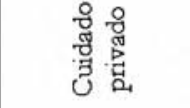 & 络 & $\mid$ & 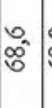 & $\hat{8}$ & ผ̂. & 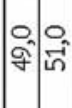 & 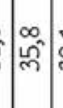 & 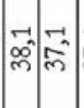 & 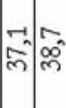 & & $\begin{array}{l}\infty \\
\dot{N} \\
\dot{N}\end{array}$ \\
\hline 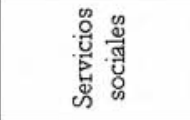 & $\stackrel{0}{2}$ & $\stackrel{\square}{\mathscr{2}}$ & $\begin{array}{l}\infty \\
\text { no } \\
\tilde{\delta}^{n}\end{array}$ & बें & 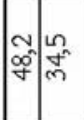 & 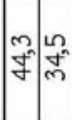 & $\begin{array}{l}n \\
\stackrel{n}{\rightarrow}\end{array}$ & 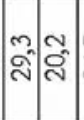 & 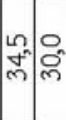 & & 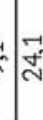 \\
\hline 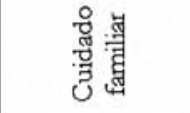 & से & $\begin{array}{l}\forall \\
\square \\
\square\end{array}$ & $\overrightarrow{0}$ & กิ่ & \begin{tabular}{|l|l}
$\infty$ & $n$ \\
in & ñ
\end{tabular} & \begin{tabular}{l|l}
$\infty$ \\
fô \\
$f^{-1}$
\end{tabular} & 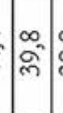 & 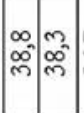 & 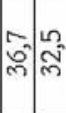 & & הิ \\
\hline & 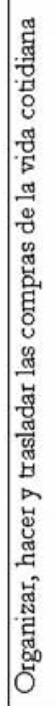 & 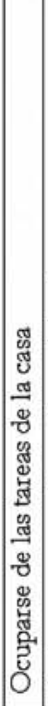 & 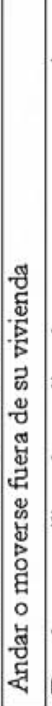 & 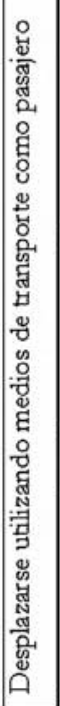 & 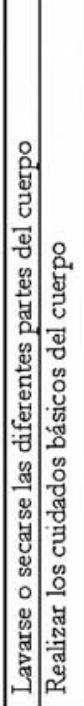 & 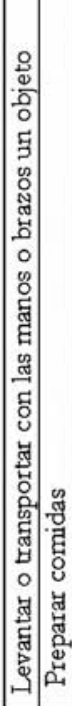 & 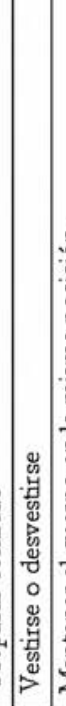 & 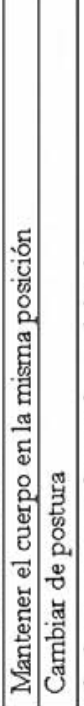 & 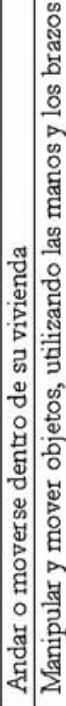 & 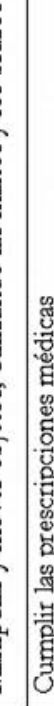 & 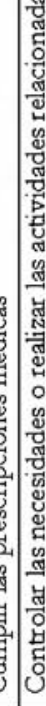 \\
\hline
\end{tabular}


que recibían no satisfacía sus necesidades. De la muestra analizada, $2873(75,8 \%)$ personas eran cuidadas en exclusiva por uno o varios familiares. Además, 286 $(7,8 \%)$ sumaba a esta ayuda el apoyo de empleadas de hogar, $136(3,4 \%)$ la de los servicios sociales y $19(0,5 \%)$ de ambos conjuntamente. 267 personas $(8,2 \%)$ disponía exclusivamente de cuidado privado y 137 (4,3\%) sólo de servicios sociales. En relación con la satisfacción de necesidades, 1039 (29\%) personas dependientes consideraba que el cuidado que recibía no satisfacía sus necesidades. Las personas con necesidades no satisfechas fueron 71 (50\%) entre quienes recibían atención sólo de servicios sociales, $754(27,3 \%)$ entre quienes recibían sólo cuidado familiar y 64 $(22,5 \%)$ entre quienes sólo contaban con cuidado privado. Las personas que combinaron el cuidado familiar con servicios sociales y mostraron necesidades no satisfechas ascendieron a $57(43,5 \%)$, a 86 $(33,3 \%)$ entre quienes combinaron cuidado familiar y privado, y a $7(41,2 \%)$ entre quienes recibieron atención de los tres proveedores.
El análisis bivariado (tabla 3) muestra que la insatisfacción de necesidades de cuidado es mayor entre las mujeres (30,6\%, frente al $24,9 \%$ de los hombres, $\mathrm{p}=0,001)$, las personas con discapacidades más severas $(40,6 \%$ entre quienes tienen 12 o más discapacidades, frente al 19,9\% de quienes tienen entre 1 y $6 ; p<0,000)$, los niveles de estudios más bajos $(29,9 \%$ de quienes tienen estudios primarios o incompletos respecto al $23 \%$ de niveles superiores; $\mathrm{p}=0,003)$, los hogares unipersonales (37\% frente al $22,1 \%$ de quienes viven en hogares de 3 ó más personas; $p<0,000$ ), con ingresos del hogar inferiores a $500 €$ (38\% frente al $23,3 \%$ ingresos por encima de $1000 € ; p<0,000)$ y quienes viven en hogares situados en municipios con más de 100.000 habitantes $(31,7 \%$ frente al $26,5 \%$ de quienes viven en hogares de menos de 10.000 habitantes; $p=0,015)$. Esta valoración negativa es mayor también entre las personas dependientes cuyos cuidadores principales: no viven en el hogar $(35,8 \%$ frente al 25,6\% de quienes conviven con su cuidador; $\mathrm{p}<0,000)$, son varones $(33,8 \%$ frente al $24,7 \%$ entre quienes reciben cuidado de una mujer; $\mathrm{p}<0,000$ ), llevan 8 ó

Tabla 2

Personas dependientes de 60 y más años que consideran que el cuidado que reciben no satisface sus necesidades, según proveedor de cuidado. España 2008

\begin{tabular}{|l|r|r|r|}
\hline & $N^{*}$ & \multicolumn{1}{|c|}{$\begin{array}{c}\text { Personas con } \\
\text { necesidades } \\
\text { no satisfechas } \\
\mathrm{N}^{*}(\%)^{* *}\end{array}$} \\
\hline Cuidado familiar & 2.873 & 75,8 & $754(27,3)$ \\
\hline Servicios sociales & 137 & 4,3 & $71(50,0)$ \\
\hline Cuidado privado & 267 & 8,2 & $64(22,5)$ \\
\hline Cuidado familiar y servicios sociales & 136 & 3,4 & $57(43,5)$ \\
\hline Cuidado familiar y cuidado privado & 286 & 7,8 & $86(33,3)$ \\
\hline Cuidado familiar, cuidado privado, servicios sociales & 19 & 0,5 & $7(41,2)$ \\
\hline Total & 3.718 & 100,0 & $1.039(29,0)$ \\
\hline
\end{tabular}

$\mathrm{N}^{*}$ Corresponde al número de observaciones reales (no ponderadas). Todos los porcentajes fueron calculados sobre la muestra ponderada. ${ }^{* *} \mathrm{chi}^{2}=55.490 ; \mathrm{p}=0,0001$. 
Tabla 3

Satisfacción insuficiente de las necesidades de atención, según variables relativas a la persona dependiente, del entorno, del cuidador y las características del cuidado

\begin{tabular}{|c|c|c|c|c|}
\hline & \multirow[t]{2}{*}{$\mathrm{N}^{*}$} & \multicolumn{2}{|c|}{$\begin{array}{c}\text { Personas con } \\
\text { necesidades no } \\
\text { satisfechas }\end{array}$} & \multirow[t]{2}{*}{$\mathrm{p}$} \\
\hline & & $\mathrm{n}^{*}$ & $\%$ & \\
\hline \multicolumn{5}{|l|}{ Sexo persona dependiente } \\
\hline Varón & 1.069 & 269 & 24,9 & \multirow[t]{2}{*}{0,001} \\
\hline Mujer & 2.649 & 770 & 30,6 & \\
\hline \multicolumn{5}{|l|}{ Edad de la persona dependiente } \\
\hline Media (desviación típica) & & & ,5) & \\
\hline Entre 60 y 74 años $(\%)$ & 1.255 & 354 & 29,0 & \multirow[t]{3}{*}{0,958} \\
\hline Entre 75 y 84 años (\%) & 1.650 & 474 & 29,2 & \\
\hline 85 y más años (\%) & 813 & 211 & 28,6 & \\
\hline \multicolumn{5}{|l|}{ Número de discapacidades } \\
\hline Media (desviación típica) & & & & \\
\hline $1-6(\%)$ & 1.253 & 238 & 19,9 & \multirow[t]{3}{*}{0,000} \\
\hline $7-11(\%)$ & 1.301 & 351 & 26,8 & \\
\hline $12+(\%)$ & 1.164 & 450 & 40,6 & \\
\hline \multicolumn{5}{|c|}{ Nivel de estudios de la persona dependiente } \\
\hline Estudios primarios o incompletos & 3.263 & 931 & 29,9 & \multirow[t]{2}{*}{0,003} \\
\hline Estudios secundarios o superiores & 455 & 108 & 23,0 & \\
\hline \multicolumn{5}{|c|}{ Tamaño del hogar(número de personas) } \\
\hline Media (desviación típica) & & & ,2) & \\
\hline 1 & 697 & 243 & 37,0 & \multirow[t]{3}{*}{0,000} \\
\hline 2 & 1.741 & 517 & 30,2 & \\
\hline 3 ó más & 1.280 & 279 & 22,1 & \\
\hline \multicolumn{5}{|l|}{ Ingresos mensuales hogar } \\
\hline Menos de 500 euros (\%) & 446 & 163 & 38,0 & \multirow[t]{4}{*}{0,000} \\
\hline De 500 a $999(\%)$ & 1.544 & 475 & 32,4 & \\
\hline Más de $1000(\%)$ & 1.517 & 348 & 23,3 & \\
\hline $\mathrm{Nc}$ & 209 & 52 & 25,5 & \\
\hline \multicolumn{5}{|l|}{ Tamaño de municipio } \\
\hline $100.000+(\%)$ & 1.350 & 415 & 31,7 & \multirow[t]{3}{*}{0,015} \\
\hline $10.001-100.000(\%)$ & 1.035 & 258 & 27,9 & \\
\hline $10.000-(\%)$ & 1.333 & 366 & 26,5 & \\
\hline \multicolumn{5}{|c|}{ Lugar de residencia del cuidador principal } \\
\hline Reside en el hogar & 2578 & 649 & 25,6 & \multirow[t]{2}{*}{0,000} \\
\hline No reside en el hogar & 1.140 & 390 & 35,8 & \\
\hline \multicolumn{5}{|l|}{ Horas de cuidado recibidas } \\
\hline Media (desviación típica) & & & & \\
\hline $0-2$ & 1.117 & 292 & 28,5 & \multirow[t]{3}{*}{0,697} \\
\hline $3-8$ & 1.190 & 321 & 27,9 & \\
\hline $9+$ & 1.261 & 373 & 29,5 & \\
\hline \multicolumn{5}{|l|}{ Sexo del cuidador principal } \\
\hline Hombre & 1.017 & 327 & 33,8 & \multirow[t]{2}{*}{0,000} \\
\hline Mujer & 2.467 & 606 & 24,7 & \\
\hline
\end{tabular}


Tabla 3

(continuación)

\begin{tabular}{|c|c|c|c|c|}
\hline \multicolumn{5}{|l|}{ Tiempo prestando los cuidados } \\
\hline Menos de 2 años & 748 & 191 & 23,9 & \multirow[t]{3}{*}{0,036} \\
\hline De 2 hasta 8 años & 1.342 & 343 & 27,4 & \\
\hline 8 y más años & 1.200 & 333 & 29,5 & \\
\hline \multicolumn{5}{|l|}{ Tiene problemas a raiz del cuidado } \\
\hline Sí tiene problemas & 2.159 & 641 & 30,8 & \multirow[t]{2}{*}{0,000} \\
\hline No ticne problemas & 451 & 49 & 12,3 & \\
\hline \multicolumn{5}{|l|}{ Relación de parentesco } \\
\hline Cónyuge o pareja & 1.278 & 362 & 28,6 & \multirow{6}{*}{0,001} \\
\hline $\mathrm{Hija} / \mathrm{o}$ & 1.413 & 371 & 28,7 & \\
\hline Nuera/yerno & 140 & 22 & 14,3 & \\
\hline Madre/padre & 4 & 3 & 100,0 & \\
\hline Hermana/o & 121 & 41 & 32,6 & \\
\hline Otra relación & 753 & 236 & 31,6 & \\
\hline \multicolumn{5}{|l|}{ Actividad económica del cuidador principal } \\
\hline Trabajando & 961 & 267 & 28,7 & \multirow[t]{4}{*}{0,025} \\
\hline En desempleo & 242 & 60 & 24,1 & \\
\hline Dedicado principalmente a labores del hogar & 845 & 196 & 23,7 & \\
\hline Otra situación & 1.227 & 356 & 29,5 & \\
\hline \multicolumn{5}{|l|}{ Nivel de estudios del cuidador principal } \\
\hline Estudios primarios o incompletos & 2.171 & 616 & 29,3 & \multirow[t]{2}{*}{0,001} \\
\hline Estudios secundarios o superiores & 1.275 & 306 & 24,0 & \\
\hline \multicolumn{5}{|l|}{ Nacionalidad del cuidador principal } \\
\hline Española & 3.248 & 894 & 28,4 & \multirow[t]{2}{*}{0,000} \\
\hline Extranjera o española y extranjera & 232 & 38 & 15,9 & \\
\hline
\end{tabular}

$\mathrm{N}^{*}$ Corresponde al número de observaciones reales (no ponderadas). Todos los porcentajes fueron calculados sobre la muestra ponderada.

más años atendiendo a la persona dependiente $(29,5 \%$ frente al $23,9 \%$ entre quienes llevan menos de 2 años; $p=0,036$ ), y en aquellos casos en los que el cuidador principal tiene problemas a raíz del cuidado $(30,8 \%$ frente al $12,3 \%$ de quienes cuyo cuidador no experimenta estos problemas; $\mathrm{p}<0,000)$.

Los modelos mostrados en la tabla 4 examinaron las variables relacionadas con la presencia de necesidades no satisfechas. El modelo 2 muestra que la probabilidad de no tener satisfechas las necesidades fue mayor entre las mujeres $(\mathrm{OR}=1,244$ sig. $=0,021)$, entre quienes experimentaron de 7 a 11 discapacidades o más de 12 respecto a quienes experimentaron 6 o menos $(\mathrm{OR}=1,566$ y $\mathrm{OR}=3,178$ respectivamente, $\mathrm{p}=0,000$ en ambos casos), y menor entre quienes vivían en hogares de 3 o más personas respecto a quienes vivían solos $(\mathrm{OR}=0,654 \mathrm{p}=0,004)$ y entre quienes vivían en hogares con 1000 euros mensuales o más respecto a los hogares con menos de 500 euros $(\mathrm{OR}=0,688$ $\mathrm{p}=0,016)$. El análisis muestra que la incorporación del factor "proveedor de cuidado" en el modelo incrementa su ajuste global de manera significativa (de $\mathrm{R}^{2}=0,89$ a $\left.\mathrm{R}^{2}=0,99\right)$. En relación con quienes recibían sólo cuidado familiar, quienes recibieron únicamente cuidado de servicios sociales y quienes recibieron cuidado familiar combinado con servicios sociales tuvieron más probabilidades de no satisfacer sus necesidades $(\mathrm{OR}=1,932 \mathrm{p}=0,001$ y $\mathrm{OR}=1,525$ $\mathrm{p}=0,039$, respectivamente). Quienes reci- 
Tabla 4

Modelo de regresión logística de los factores asociados con necesidades de atención no satisfechas. España 2008

\begin{tabular}{|c|c|c|c|c|}
\hline \multirow{2}{*}{$\begin{array}{l}\text { Satisfacción de necesidades (ref: el cuidado que recibe } \\
\text { satisface sus necesidades) }\end{array}$} & \multicolumn{2}{|c|}{ Modelo 1} & \multicolumn{2}{|c|}{ Modelo 2} \\
\hline & $\mathrm{p}$ & $\operatorname{Exp}(\mathrm{B})$ & $\mathrm{p}$ & $\operatorname{Exp}(\mathrm{B})$ \\
\hline Sexo (ref: hombre) & 0,016 & 1,253 & 0,021 & 1,244 \\
\hline Severidad de la discapacidad (ref: $1-6$ discapacidades) & 0,000 & & 0,000 & \\
\hline $7-11$ & 0,000 & 1,584 & 0,000 & 1,566 \\
\hline $12+$ & 0,000 & 3,243 & 0,000 & 3,178 \\
\hline Edad de la persona dependiente (ref: $60-74$ ) & 0,038 & & 0,052 & \\
\hline $75-84$ & 0,210 & 0,888 & 0,217 & 0,888 \\
\hline $85+$ & 0,011 & 0,744 & 0,015 & 0,752 \\
\hline $\begin{array}{l}\text { Nivel de estudios de la persona dependiente (ref: estudios } \\
\text { primarios o incompletos) }\end{array}$ & 0,072 & 0,789 & 0,124 & 0,815 \\
\hline Tamaño del hogar (ref: I persona) & 0,001 & & 0,003 & \\
\hline 2 & 0,165 & 0,845 & 0,382 & 0,896 \\
\hline 3 ó más & 0,001 & 0,621 & 0,004 & 0,654 \\
\hline Ingresos mensuales hogar (ref: menos de 500 euros) & 0,011 & & 0,020 & \\
\hline De 500 a 999 & 0,449 & 0,904 & 0,536 & 0,920 \\
\hline 1000 ó más & 0,009 & 0,669 & 0,016 & 0,688 \\
\hline No contesta & 0,068 & 0,666 & 0,098 & 0,690 \\
\hline Tamaño de municipio (ref: $100.000+$ ) & 0,010 & & 0,011 & \\
\hline $10.001-100.000$ & 0,030 & 0,809 & 0,031 & 0,809 \\
\hline $10.000-$ & 0,004 & 0,751 & 0,005 & 0,753 \\
\hline Horas diarias de cuidado recibidas (ref: $0-2$ ) & 0,058 & & 0,258 & \\
\hline $3-8$ & 0,251 & 0,887 & 0,685 & 0,957 \\
\hline $9+$ & 0,018 & 0,768 & 0,124 & 0,839 \\
\hline Constante & 0,000 & 0,456 & 0,000 & \\
\hline \multicolumn{5}{|c|}{ N: 3.566. Chi-cuadrado: $206,827 p<0,001 . R^{2}$ de Nagelkerke: 0,089} \\
\hline \multicolumn{5}{|l|}{ Proveedor de cuidado (ref: cuidado familiar) } \\
\hline Servicios sociales & & & 0,001 & 1,932 \\
\hline Cuidado privado & & & 0,015 & 0,673 \\
\hline Cuidado familiar y servicios sociales & & & 0,039 & 1,525 \\
\hline Cuidado familiar y cuidado privado & & & 0,745 & 1,050 \\
\hline Cuidado familiar, privado y servicios sociales & & & 0,198 & 2,035 \\
\hline Constante & & & 0,000 & 0,399 \\
\hline
\end{tabular}

bieron únicamente cuidado privado tuvieron más probabilidades de satisfacer sus necesidades que quienes recibieron sólo cuidado familiar $(\mathrm{OR}=0,673 \mathrm{p}=0,015)$. Asimismo, se realizaron diferentes modelos (no mostrados en este trabajo) cambiando únicamente las categorías de referencia para la variable "proveedor de cuidado". En ellos se observó: (1) que las personas que recibieron sólo cuidado privado tuvieron significa- tivamente más probabilidades de satisfacer sus necesidades que el resto de proveedores; (2) que quienes recibieron sólo cuidado de servicios sociales tuvieron más probabilidades de no satisfacer sus necesidades, en relación con quienes recibieron sólo cuidado privado, sólo cuidado de la familia o cuidado familiar combinado con cuidado privado. 


\section{DISCUSIÓN}

Este trabajo constituye el primer estudio con representatividad nacional sobre factores relacionados con las necesidades de cuidado no satisfechas de las personas mayores no institucionalizadas en España, y en particular sobre el efecto del proveedor de cuidado en dicha satisfacción. De acuerdo con nuestros resultados, el porcentaje de personas de $60 \mathrm{y}$ más años que considera que la atención que recibe no satisface sus necesidades es de un $29 \%$. Estudios precedentes han estimado que entre el $2 \%$ y el $35 \%$ de las personas mayores dependientes que viven en la comunidad tienen necesidades no cubiertas en Estados Unidos, dependiendo de qué se incluya en la definición de necesidad ${ }^{3}$. Por su parte, Allen y Mor ${ }^{14}$ establecieron la prevalencia de necesidades no cubiertas en un 44\% entre población adulta necesitada de ayuda, si se incluían actividades del hogar complejas. En el ámbito español, Tomás et al. ${ }^{21}$ hallaron que la prevalencia de necesidades no cubiertas para las actividades del cuidado personal en personas dependientes no institucionalizadas mayores de 74 años en una Zona de Salud de Zaragoza era del $22,1 \%$. El trabajo de Otero et al. ${ }^{22}$ encontró que el $40 \%$ de los mayores de 64 años de una muestra representativa de Leganés, Madrid, tenía alguna necesidad no cubierta.

Nuestros resultados indican que el proveedor de cuidado tiene una relación estadísticamente significativa con la satisfacción de las necesidades de atención de las personas mayores dependientes. El cuidado familiar es el tipo de atención mayoritaria. Sin embargo, nuestro análisis sugiere que la familia no es el agente más eficaz para cubrir sus necesidades. Los resultados apuntan a que el cuidado privado en exclusiva es el que más probabilidades presenta de satisfacer las necesidades de las personas mayores dependientes. En este sentido, investigaciones precedentes han puesto de manifiesto que el grado de parentesco no asegura capacitación para tratar con las ramificaciones físicas y psicológicas de la provisión de cuida- $\operatorname{dos}^{23}$. Asimismo, estudios cualitativos ${ }^{24}$ han sugerido mayores ventajas del cuidado formal sobre el informal. En estos, aquellas personas dependientes cuya relación con el cuidador era principalmente contractual veían bien cubiertas sus necesidades, se sentían cómodos y percibían control sobre su propia vida. Las personas dependientes cuya relación con el cuidador se basaba en la buena voluntad con frecuencia sentían vergüenza y frustración. Las consecuencias de percibirse como una carga ${ }^{25}$ podrían ser la causa de la insatisfacción con el cuidado recibido por parte de familiares. Estos resultados sugieren que la percepción de la persona dependiente sobre la situación de su cuidador influye significativamente en la satisfacción con el cuidado, tal y como ha sido expuesto en investigaciones precedentes ${ }^{26}$. El trabajo apunta también a una limitada satisfacción de necesidades por parte de los servicios públicos, especialmente cuando se trata del único agente proveedor.

Aparte del proveedor de cuidado, este trabajo ha identificado algunos factores relevantes relacionados con una cobertura de cuidados insatisfactoria. Ser mujer, sufrir una discapacidad más severa, tener entre 60 y 74 años (respecto a los mayores de 84), la soledad residencial, los ingresos reducidos y vivir en grandes ciudades se relaciona con mayores probabilidades de tener necesidades de atención insatisfechas. Estos hallazgos son consistentes con la mayoría de los encontrados en estudios similares. Respecto al sexo, Allen ${ }^{14}$ halló que las mujeres tenían mayor probabilidad de tener necesidades de cuidado no cubiertas para las AIVD. En el contexto español, Tomás et al. ${ }^{21}$ encontraron que ser mujer se relacionaba con una mayor probabilidad de tener necesidades de cuidado no cubiertas, aunque su resultado no controló el número de miembros del hogar. En lo referente a la severidad de la discapacidad, las necesidades de cuidado insatisfechas son mayores entre quienes tienen presentan un mayor número de discapacidades $^{14,16,27}$. En este sentido, la insatisfacción con el cuidado es mayor entre quienes experimentan dolor y ansiedad ${ }^{24}$. Nuestros resulta- 
dos en relación con la edad son coincidentes con estudios anteriores ${ }^{14}$. A este respecto, se ha sugerido que los adultos jóvenes tienen expectativas más elevadas sobre su propio cuidado, debido a que están acostumbrados a mayores estándares de protección pública, lo que les lleva a estar menos satisfechos con la atención recibida ${ }^{13}$. Sobre la relación entre la insatisfacción con el cuidado y el nivel educativo de las personas dependientes, los estudios realizados ofrecen resultados divergentes ${ }^{13}$. Trabajos en EEUU sobre población de 70 y más años hallaron que las necesidades de cuidado no cubiertas son mayores entre los niveles educativos inferiores ${ }^{16}$. No obstante, en España, Otero et al. ${ }^{22}$ no hallaron relación entre el nivel educativo y las necesidades de cuidado no cubiertas tras controlar otras variables. En ambos estudios se tuvieron en cuenta indicadores sobre ingresos.

Las formas de convivencia de las personas mayores dependientes son claves en la satisfacción de sus necesidades de cuidado ${ }^{28} \mathrm{y}$ en su bienestar ${ }^{29}$. Existen evidencias de que las personas que viven solas tienen más probabilidades de no tener cubiertas sus necesidades de cuidado $^{3,16,21}$. En este sentido, Allen y Mor ${ }^{14}$ encontraron que las necesidades de cuidado no cubiertas eran mayores a medida que se reducía el número de personas con las que podía contar la persona dependiente. Nuestros resultados también confirman que una mejora en los ingresos del hogar puede ser un facilitador de la satisfacción de necesidades, en concordancia con lo hallado por Langa y Martínez ${ }^{30}$. En relación con el tamaño del municipio, estudios previos encontraron, para el caso de España, que las personas dependientes que viven en zonas rurales tienen más probabilidades de recibir cuidado familiar y menos cuidado formal que quienes viven en entornos urba$\operatorname{nos}^{12}$, y que en estas áreas las tasas de discapacidad son algo más elevadas y se dan situaciones de dependencia más severas ${ }^{31}$. En Canadá se han encontrado carencias significativas en la provisión de servicios de cuidado a personas dependientes en las zonas rurales ${ }^{32}$.
La presente investigación tiene limitaciones que deben considerarse al interpretar sus resultados. En primer lugar, la muestra incluyó únicamente aquella personas dependientes que respondían ellas mismas la encuesta. En consecuencia, es probable que la muestra escogida no sea representativa en términos de severidad de la discapacidad, ya que quienes no pudieron responder directamente a la encuesta no están representados en el análisis efectuado. En segundo lugar, la variable dependiente utilizada no permite identificar qué necesidades específicas (materiales, de comunicación, afectivas, etc.) son las que la persona dependiente no encuentra suficientemente satisfechas. En este sentido, sería conveniente dilucidar si existe relación, y de qué índole, entre el tipo de necesidades de atención (que implican unas determinadas actividades de cuidado) y la elección o disponibilidad de unos u otros proveedores. El conocimiento de esta relación contribuiría a explicar los mecanismos de satisfacción de las necesidades de las personas mayores dependientes. Por último, sería conveniente investigar en mayor profundidad la satisfacción de necesidades en los diferentes proveedores de cuidado por separado, con el fin de conocer el efecto de variables más específicas de cada uno de ellos; por ejemplo, sería relevante indagar, para personas que reciben sólo cuidado familiar o de empleadas de hogar, el efecto de la corresidencia entre cuidador y receptor en la satisfacción de necesidades.

A pesar de sus limitaciones, los hallazgos de este trabajo son consistentes y tienen implicaciones relevantes para el diseño de políticas públicas. Nuestros resultados ponen en duda la eficacia de las medidas de potenciación de la familia como agente más apropiado para cubrir esas necesidades y sugieren posibles aspectos positivos del cuidado privado, principalmente provisto por empleadas de hogar y, generalmente, en condiciones de precariedad e informalidad ${ }^{11}$. A este respecto sería conveniente un mayor control por parte de las administraciones públicas sobre la calidad de 
este tipo de atención y sobre las relaciones laborales en las que se fundamenta. Asimismo y a la luz de los datos, las administraciones públicas deberían tener como objetivo prioritario aumentar la eficacia de los servicios de cuidado financiados con fondos públicos.

\section{AGRADECIMIENTOS}

Queremos agradecer al Portal Mayores (CSIC-IMSERSO), en particular a Antonio Abellán y Cecilia Esparza, el trabajo de preparación del fichero de datos con el que se ha realizado el análisis estadístico.

\section{BIBLIOGRAFÍA}

1. European Commission. 2009 ageing report: economic and budgetary projections for the EU-27 Member States (2008-2060). Bruselas: European Commission; 2009

2. Mestheneos E, Triantafillou J. Supporting family carers of older people in Europe-the Pan-European background. Hamburgo: Eurofamcare; 2005.

3. Williams J, Lyons B, Rowland D. Unmet Long-Term Care Needs of Elderly People in the Community; A Review of the Literature. Home Health Care Serv Q. 1997; 16(1):93-119.

4. Boletín Oficial del Estado. Ley 39/2006, de 14 de diciembre, de Promoción de la Autonomía Personal y Atención a las personas en situación de dependencia. BOE núm 299 de 15-12-2006.

5. Abellán García A, Esparza Catalán C. Las personas mayores con dependencia. En Puyol Antolín R, Abellán García A editores. Envejecimiento y dependencia, una mirada al panorama futuro de la población española. Madrid: Mondial Assistance; 2006.p.55-72.

6. Fillenbaum GG. Activities of daily living. En: Maddox G, editor. The Encyclopedia of Aging. Second edition. New York: Springer Publishing Company; 1995.p. 7-9.

7. Willmot P. Social Networks, Informal Care, and Public Policy. London: Policy Studies Institute; 1986.

8. Harlton SV, Fast J, Keating N. Defining eldercare for Policy and Practice: Perspectives Matter. Fam Relat. 1998; 47(3):281-8.

9. IMSERSO. Libro Blanco de la Dependencia. Madrid: Instituto de Mayores y Servicios Sociales; 1995.
10. Blandford A, Chappell N. Informal and formal care: exploring the complementarity. Ageing Soc. 1991; 11(3):299-317.

11. IMSERSO. Cuidado a la dependencia e inmigración. Madrid: Ministerio de Trabajo y Asuntos Sociales; 2005.

12. Rogero-García J. Distribución en España del cuidado formal e informal a las personas de 65 y más años en situación de dependencia. Rev Esp Salud Pública. 2009; 83(3): 393-405.

13. Sitzia J, Wood N. Patient satisfaction: a review of issues and concepts. Soc Sci Med. 1997; 45(12):1829-43.

14. Allen SM, Mor V. The Prevalence and Consequences of Unmet Need Contrasts Between Older and Younger Adults with Disability. Med Care. 1997; 35(11):1132-48.

15. Lai DWL. Impact of culture on depressive symptoms of elderly Chinese immigrants, Canadian J Psychiatry. 2004; 49(12):820-7.

16. Desai MM, Lentzner HR, Weeks JD. Unmet Need for Personal Assistance With Activities of Daily Living Among Older Adults. Gerontologist. 2001; 41(1):82-8.

17. INE. Encuesta de Discapacidad, Autonomía personal y situaciones de Dependencia 2008. Metodología. Madrid: Instituto Nacional de Estadística; 2010.

18. Todorov A, Kirchner C. 'Bias in Proxies' Reports of Disability Data From the Nacional Health Interview Survey on Disability. Am J Public Health. 2000; 90(8):1248-1253.

19. Harrel FE Regression modeling strategies with applications to linear models logistic regression and survival analysis. New York: Springer; 2001.

20. Norusis M SPSS 13.0. Advanced Statistical Procedures Companion. New Jersey: Prentice Hall; 2005.

21. Tomás Aznar C, Moreno Aznar L, Germán Bes C, Alcalá Nalváiz T, Andrés Esteban E. Dependencia y necesidades de cuidados no cubiertas de las personas mayores de una zona de salud de Zaragoza. Rev Esp Salud Pública. 2002; 76(3):215-26

22. Otero A, García de Yebenes M, Rodriguez-Laso A, Zunzunegui M. Unmet home care needs among community-dwelling elderly people in Spain. Aging Clin Exp Res. 2003; 15(3):234-42. 
23. Riess-Sherwood P, Given BA, Given ChW. Who Cares for the Caregiver: Strategies to Provide Support. Home Health Care Manag Pract. 2002; 14(2):110-21.

24. Galvin R. Challenging the Need for Gratitude: Comparisons Between Paid and Unpaid Care for Disabled People. J Sociol. 2004; 40(2):137-55.

25. Matthias RE, Benjamin AE. Paying Friends, Family Members, or Strangers to Be Home-Based Personal Assistants. Journal of Disability Policy Studies. 2008; 18(4), 205-18.

26. Bauld L, Chesterman J, Judge K. (2000). Measuring satisfaction with social care amongst older service users: issues from the literature. Health Soc Care Community. 2000; 8(5):316-24.

27. Geron SM, Smith K, Tennstedt S, Jette A, Chassler D, Kasten L. The Home Care Satisfaction Measure: A Client-Centered Approach to Assessing the Satisfaction of Frail Older Adults With Home Care Services. J Gerontol B Psychol Sci Soc Sci. 2000; 55B(5): S25970.

28. Ahmed Mohamed K; Rojo Pérez F; Fernández Mayoralas G, Prieto Flores ME, Rodríguez Rodríguez V, Lardiés Bosque R. Red familiar y distancia afectiva en la calidad de vida de la población mayor. En: Despoblación, envejecimiento y territorio: un análisis sobre la población española. León: Universidad de León; 2009.p. 375-86.

29. Rojo-Pérez F, Fernández-Mayoralas G, Forjaz MJ, Delgado-Sanz MC, Ahmed Mohamed K, MartínezMartín P, Prieto-Flores ME, Rojo-Abuín JM. Población mayor, Calidad de Vida y redes de apoyo: demanda y prestación de cuidados en el seno familiar. Madrid: Escuela Nacional de Sanidad, Instituto de Salud Carlos III-Ministerio de Ciencia e Innovación; 2009.

30. Langa D, Martínez D. Redes familiares, cuidados y clases sociales en Andalucía. Madrid: Alianza; 2009.

31. Puga MD, Abellán A. El proceso de discapacidad. Un análisis de la Encuesta sobre Discapacidades, Deficiencias y Estado de Salud, 1999. Madrid: Fundación Pfizer; 2004.

32. Morgan DG, Semchuk KM, Stewart NJ D’Arcy C. Rural families caring for a relative with dementia: barriers to use of formal services. Soc Sci Med. 2002; (55):1129-42. 